Journal of Acute Disease

journal homepage: www.jadweb.org

\title{
Hypertriglyceridemia-induced acute pancreatitis: a systematic review of the literature
}

Ionut Negoi ${ }^{1 *}$, Sorin Paun ${ }^{1}$, Massimo Sartelli ${ }^{2}$, Fausto Catena ${ }^{3}$, Luca Ansaloni ${ }^{4}$, Ruxandra Irina Negoi ${ }^{5}$, Mircea Beuran ${ }^{1}$

${ }^{I}$ General Surgery Department, Emergency Hospital of Bucharest, Carol Davila University of Medicine and Pharmacy Bucharest, Bucharest, Romania

${ }^{2}$ Department of Surgery, Macerata Hospital, Via Santa Lucia 2, 62019, Macerata, Italy

${ }^{3}$ Department of Surgery, Maggiore Hospital, Parma, Italy

${ }^{4}$ General Surgery Department, Papa Giovanni XXIII Hospital, Bergamo, Italy

${ }^{5}$ Anatomy Department, Carol Davila University of Medicine and Pharmacy, Bucharest, Romania

\section{ARTICLE INFO}

Article history:

Received 25 Aug 2016

Received in revised form 18 Sep 2016

Accepted 22 Oct 2016

Available online 8 Dec 2016

\section{Keywords:}

Hypertriglyceridemia-induced

Acute pancreatitis

Systematic review

Etiology

\begin{abstract}
Objective: To summarize the current evidence about the hypertriglyceridemia-induced acute pancreatitis (HAP).

Methods: Systematic review of the English language literature was conducted using PubMed/ Medline database from its inception until August 2016. As a searching methodology, we have used a combination of 'acute pancreatitis' and 'hypertriglyceridemia' as keywords into the title. Results: The diagnosis of HAP should be based on two out of the three criteria recommended by the international guidelines: characteristic clinical picture, serum pancreatic enzymes, and appropriate imagistics. The diagnosis of HAP should be distinguished between mild hypertriglyceridemia ( $>150 \mathrm{mg} / \mathrm{dL}$ ), which accompanies around one-third of all-causes acute pancreatitis, and severe hypertriglyceridemia $(>1000 \mathrm{mg} / \mathrm{dL})$ which generates acute pancreatitis. There is mixed evidence regarding a worse prognosis for patients with HAP, and a clear conclusion cannot be drawn. Similar to all the other etiologies, in HAP the initial treatment efforts should be nonspecific and addressed to acute pancreatitis, while pharmacologic and mechanical techniques should be added to lower the serum triglycerides as soon as possible.

Conclusions: We may conclude that HAP should be managed to respect all the general principles, also adding all the available resources to lower the serum triglycerides value, as early as possible in the acute setting and on long-term to prevent recurrences.
\end{abstract}

\section{Introduction}

Hypertriglyceridemia is the third most frequent cause of acute pancreatitis after alcohol consumption and gallstone disease, with a reported frequency of $1 \%-10 \%$ of cases of acute pancreatitis[1-3]. The risk of acute pancreatitis is about $5 \%$ at triglyceride levels of over $1000 \mathrm{mg} / \mathrm{dL}$, and $10 \%-20 \%$ for values of more than 2000 $\mathrm{mg} / \mathrm{dL}[4]$. On the other hand, increased cholesterol levels are not associated with acute pancreatitis[5]. Dyslipidemia is defined as values of total cholesterol, low density lipoprotein-cholesterol, triglycerides, apolipoprotein-B, and lipoprotein (a) greater than acute biliary as values of high density lipoprotein-cholesterol or apolipoprotein A lower the 10th percentile of the general

\footnotetext{
*Corresponding author: Ionut Negoi, Senior Lecturer of Surgery, General Surgery Department, Emergency Hospital of Bucharest, Carol Davila University of Medicine and Pharmacy Bucharest, Bucharest, Romania

Tel: +40215992308

E-mail: negoiionut@gmail.com

The journal implements double-blind peer review practiced by specially invited international editorial board members.
}

population[6,7]. The pathophysiological mechanisms include hydrolysis of the triglycerides by pancreatic lipase, with excessive formation of free fatty acids, secondary inflammatory changes and capillary injuries[3]. Blood hyperviscosity with tissue ischemia plays an additional role.

The objective of the present study is to summarize the current evidence about the hypertriglyceridemic acute pancreatitis.

\section{Materials and methods}

This systematic review of the English language literature was conducted using PubMed/Medline database from its inception until August 2016. As searching methodology, we have used a combination of 'acute pancreatitis' and 'hypertriglyceridemia' as keywords into the title. We conducted the latest literature search in 23rd August 2016.

\section{Results}

The electronic search revealed 84 articles, out of which 31 fulltext papers were included in the current review (Figure 1). 


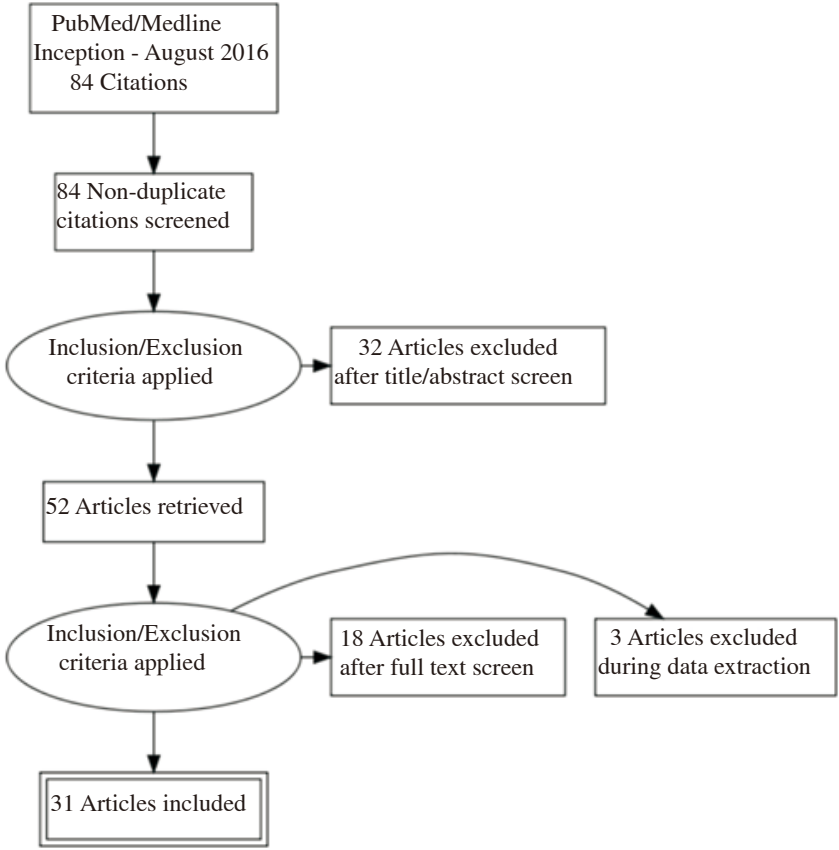

Figure 1. PRISMA flow diagram.

\subsection{Diagnosis}

According to the current guidelines, the diagnosis of acute pancreatitis should be based on two out of the three criteria: (a) characteristic abdominal pain, with acute onset, severe, persistent, radiating to the back; (b) serum lipase or amylase $>$ three times the upper normal value; (c) characteristic findings on computed tomography or magnetic resonance imaging or ultrasonography[8,9]. Although the current evidence presents hyperlipemia as etiology for acute pancreatitis, there is no cut-off value shown to indicate hypertriglyceridemia as a cause in a particular patient. According to this, the clinician should conduct the appropriate workup to exclude other possible etiologies, while certifying an increased value for serum lipids (Figures 2 and 3, unpublished results of [10]).
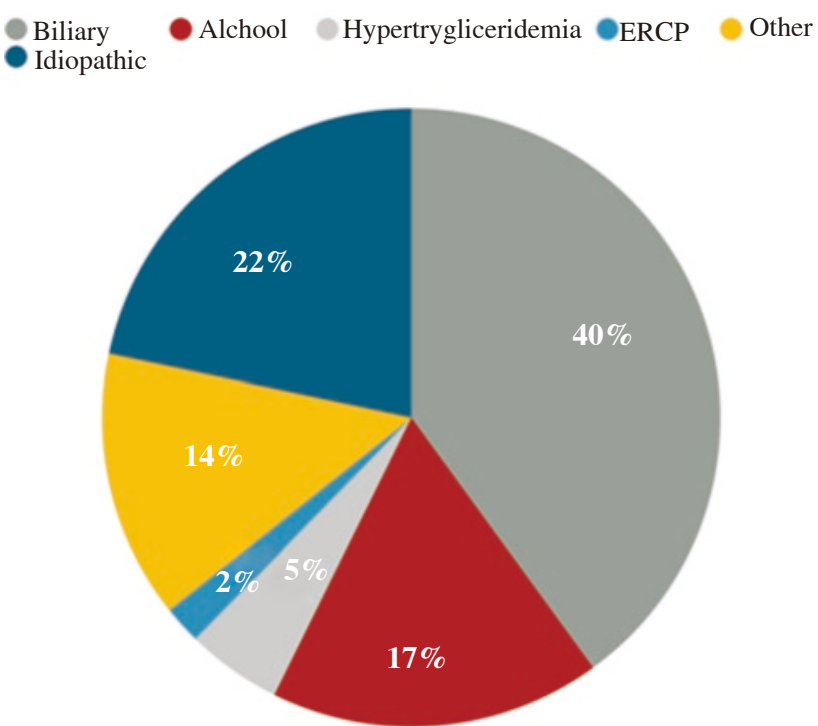

Figure 2. The etiology of acute pancreatitis in 624 patients managed in Emergency Hospital of Bucharest during four years. It can be observed that hypertrig lyceridemia accounts for $5 \%$ of cases[10].

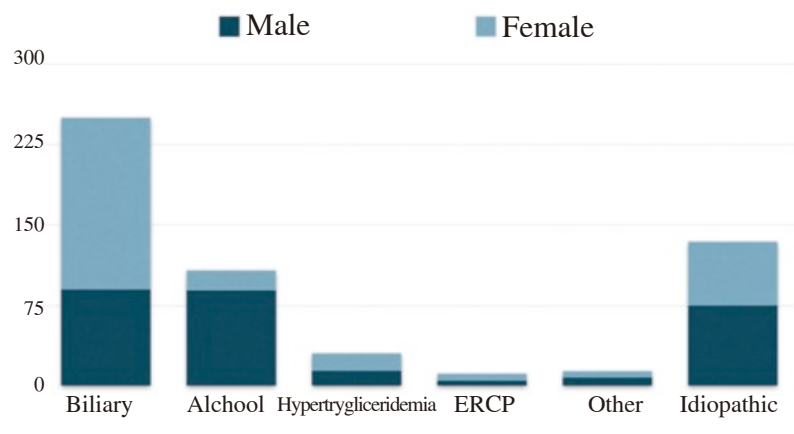

Figure 3. The etiology of acute pancreatitis in 624 patients managed in Emergency Hospital of Bucharest during four years. It can be observed that hypertriglyceridemia was found more frequent in female patients[10].

\subsection{Prognosis worse than other etiologies: heterogeneous evidence}

Although the clinical picture of hypertriglyceridemic acute pancreatitis is similar to the other etiopathology forms, it seems that its severity may be accentuated[11]. Tai et al. analyzed 126 cases of hypertriglyceridemia-induced acute pancreatitis (HAP) and 168 patients with acute biliary pancreatitis[12]. They concluded that the rate of severe and recurrent acute pancreatitis was higher in patients with hypertriglyceridemia (31.0\% vs. $26.2 \%)$, as were the comorbidities associated with diabetes mellitus (20.6\% vs. $1.6 \%$ ) [12]. Although the proportion of gastrointestinal bleeding, sepsis, and multiple organ failure was lower in patients with HAP, no differences regarding mortality were observed[12]. However, a systematic review conducted by Carr et al., having a primary objective to determine if hypertriglyceridemia induces a more severe acute pancreatitis found heterogeneous and inconsistent results[13].

\subsection{Treatment of the acute episode-general principles for acute pancreatitis, adding combined efforts to decrease the serum triglycerides}

Similar to all the other etiologies, in HAP the initial treatment efforts should be nonspecific and addressed to acute pancreatitis, while pharmacologic and mechanical techniques should be added to lower as soon as possible the serum triglycerides (see Figure 4[14]). A low-fat diet is required to decrease the triglyceride value, the enteral nutrition being the preferred way when feasible. Although an omega-3 rich diet may influence the inflammatory response in critically ill patients, their impact on triglycerides during acute illness is unknown[15]. Plasmapheresis may be considered in patients with HAP, although a significant clinical benefit was not clearly proven. In most patients, plasmapheresis produces a $66.3 \%$ decrease in triglycerides after one session, and $83.3 \%$ after two sessions[16]. Chen et al. assessed the results plasmapheresis after the introduction of this procedure in their hospital, comparing 60 patients with HAP with a control group of 34 patients[17]. The authors observed no difference in overall mortality and morbidity with plasmapheresis, concluding that timing of this procedure, ideally as soon as possible, may provide superior results[17]. A retrospective study of HAP during pregnancy compares five patients who benefit from plasmapheresis 
with five cases which not[18]. Plasmapheresis decreased the inflammatory response from $100 \%$ to $28.6 \%$, and the hospitalization time from $(37.0 \pm 20.8)$ days to $(17.3 \pm 6.7)$ days[18]. According to the guideline of therapeutic apheresis in clinical practice proposed by the American Society for Apheresis, plasma exchange in hypertriglyceridemic acute pancreatitis has a weak recommendation, based on the evidence coming from no randomized controlled trials, one controlled trial, 12 case series, and 33 case reports[19]. In these patients, the therapeutic plasma exchange may be done using both, centrifugal and double membrane filtration techniques. It seems that centrifugal methods have a higher efficacy, due to occlusion of the filters by triglycerides in the double membrane method[19].

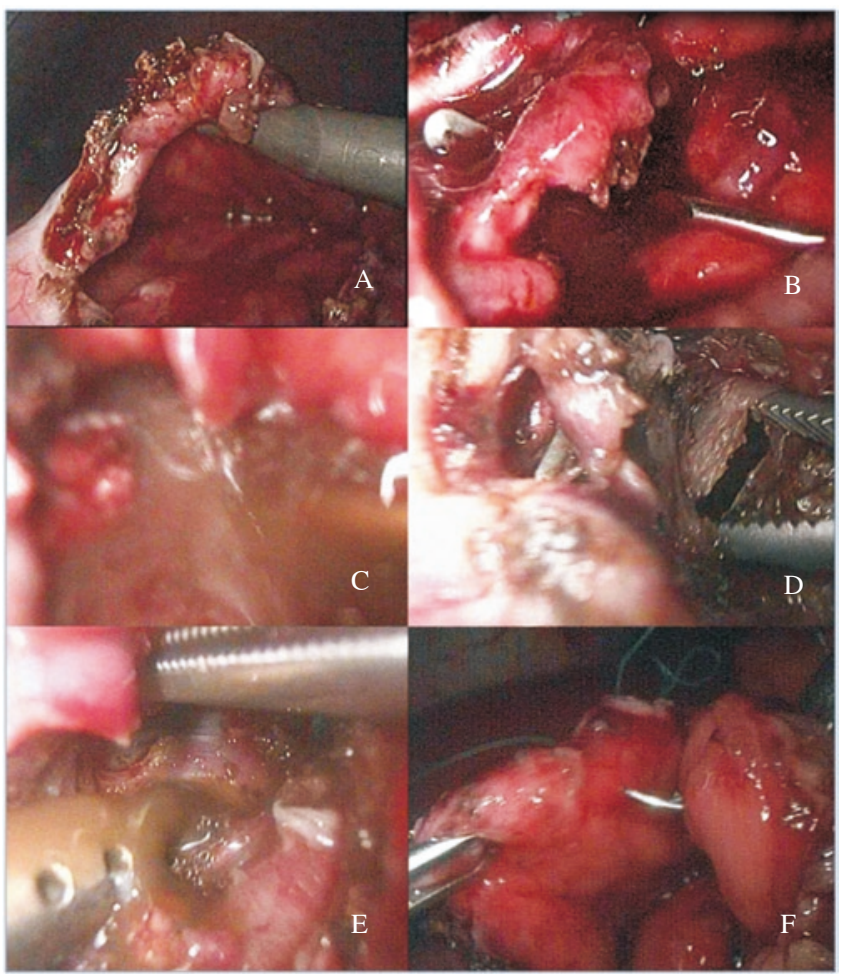

Figure 4. Intraoperative aspect of a laparoscopic anterior transgastric pseudocystogastroanatomosis, for a giant pancreatic pseudocyst, four months after a severe $\mathrm{HCP}[14]$.

A: Anterior gastric wall incision; B: Transgastric puncture of the pseudocyst; C: Posterior gastric wall incision with pseudocyst's content evacuation; D: Pseudocyst's content aspiration; F: Anterior gastric wall running suture.

\subsection{Prevention of recurrences-the serum triglyceride value should be lower than $1000 \mathrm{mg} / \mathrm{dL}$}

According to the National Cholesterol Education Program, the hypertriglyceridemia degrees of severity can be defined as: a) moderately elevated 150-199 mg/dL; b) increased: 200-499 mg/dL; and very high: $\geqslant 500 \mathrm{mg} / \mathrm{dL}[20]$. The current evidence suggests that pharmacological treatment of hypertriglyceridemia with fibrates, fish oil, and nicotinic acid should be started for triglyceride levels above $1000 \mathrm{mg} / \mathrm{dL}$ to prevent acute pancreatitis[7]. Along with the decrease in fat and carbohydrates food intake, the fibrates are recommended as first-line therapeutic agents in acute pancreatitis prevention[21]. The medical control of triglycerides values, with a reduction of those below $500 \mathrm{mg} / \mathrm{dL}$ can prevent the recurrence of acute pancreatitis[4].

\section{Discussion}

In patients with acute pancreatitis it is imperative to distinguish between mild hypertriglyceridemia (> $150 \mathrm{mg} / \mathrm{dL}$ ), which accompanies $34 \%-47 \%$ of all causes acute pancreatitis, and severe hypertriglyceridemia (> $1000 \mathrm{mg} / \mathrm{dL}$ ) which generates acute pancreatitis[13]. Although there is no cut-off value to indicate hypetrigliceridemia as etiology for acute pancreatitis, the mean serum triglycerides value was $(2654 \pm 2451) \mathrm{mg} / \mathrm{dL}$ in the systematic review of Carr et al.[13]. Murphy et al. studied the incidence of acute pancreatitis due to hypertriglyceridemia in the population living between 1993-2007 in Tayside, Scotland, with a follow-up of 15 years[22]. According to the highest triglyceride value, the study population was divided in three groups: $(1)<149$ $\mathrm{mg} / \mathrm{dL}$; (2) 150-499 mg/dL; and (3) > $500 \mathrm{mg} / \mathrm{dL}$. After covariate adjustment and removal of patients with gallstones, alcohol-related comorbidities, chronic pancreatitis, renal failure, the risk of incident HAP increased by $4 \%$ for every $100 \mathrm{mg} / \mathrm{dL}$ increase in triglyceride concentration[22].

Although a life-threating complication, fortunately, the hypertriglyceridemia is a rare cause of acute pancreatitis in pregnant women, usually occurring during the last two trimester of pregnancy[23,24]. Huang et al. reviewed 21 pregnant women diagnosed with acute pancreatitis, $95 \%$ being in the third trimester of gestation[18]. Compared to acute biliary pancreatitis, the hypertriglyceridemic one was more frequent (14\% vs. $48 \%$ ) and more severe $(0 \%$ vs. $40 \%)$ [18]. The mean serum values for triglycerides was $(1664.6 \pm 984.9) \mathrm{mg} / \mathrm{dL}$ in moderately severe acute pancreatitis and $(2701.7 \pm 2141.5) \mathrm{mg} / \mathrm{dL}$ in the severe form of the disease $(P>0.05)[18]$.

Tamoxifen, a selective estrogen receptor modulator, can have paradoxical effects on the lipid metabolism, with significant increases in the triglyceride and chylomicron levels[25-27]. When patient's risk for HAP are increased, letrozole may be used, knowing that it has no effects on the serum lipids level[28]. Everolimus, a mechanistic target of rapamycin inhibitor usually used to treat metastatic pancreatic neuroendocrine tumors and renal cell carcinoma, and for post-transplant immunosuppression was described to induce severe hypertriglyceridemia and acute pancreatitis[29]. In human immunodeficiency virus-positive patients, who develop hypertriglyceridemia secondary to retroviral therapy, the risk of acute pancreatitis is low[30]. In this study the incidence of acute pancreatitis was 1.2-4.9/1000 person-years when triglycerides were $<1500 \mathrm{mg} / \mathrm{dL}$, and $0 / 1000$ person-years when triglycerides were $>1500 \mathrm{mg} / \mathrm{dL}[30]$.

Although inconclusive with increasing evidence, there are studies which suggest that prognosis of HAP is more severe than for other causes of acute pancreatitis[31]. Rashid et al. analyzed 5500 patients with severe hypertriglyceridemia that accessed health care resources during 6.5 years and found a frequency for acute pancreatitis of 5.4\%[32]. Mean healthcare costs during the first year after hypertriglyceridemia diagnosis were $\$ 25343 \pm 33139$ for patients with acute pancreatitis, compared with $\$ 15195 \pm 24040$ for patients without acute pancreatitis[32]. On the other hand, in patients with all causes acute pancreatitis, hypertriglyceridemia is associated with a 
higher rate of hypocalcemia ( $86.7 \%$ vs. $63.5 \%, P<0.01), 24$ h Acute Physiology and Chronic Health Evaluation II score (13.6 \pm 5.7 vs. $10.7 \pm 4.6, P<0.01)$, shock (37.9\% vs. $14.5 \%, P<0.01)$, infection (37.4\% vs. $18.3 \%, P<0.01)$, and mortality $(13.1 \%$ vs. $9.1 \%, P<$ 0.01)[33]. Hypertriglyceridemia is also an independent risk factor for acute kidney injury[34]. The hypothesis behind this is that the action of pancreatic enzymes on triglycerides accumulated in the kidneys may explain acute renal injury in the early phase of the disease[34]. Another negative impact of excess fatty acids in pancreatic acinar cells seems to be on aggravation the expression of genes involved endoplasmic reticulum stress-induced unfolded protein response[35]. An et al. found as potential biomarkers for disease severity the upregulation of miR24-3p, 361-5p, 1246 and 222-3p, and the downregulation of miR181a-5p $(P<0.05)[36]$. These microRNAs showed a good area under the curve for evaluation of the disease severity[36].

Addressing treatment of HAP, a study published in Pancreas journal this year concluded that double filtration plasma apheresis decreases the in-hospital stay for patients with severe hypertriglyceridemia associated acute pancreatitis from 10 to 5 days $(P=0.009)[37]$. In the subgroup of patients with triglycerides $>5000 \mathrm{mg} / \mathrm{dL}$, the plasmapheresis decreased the hospitalization from 11 to 5 days $(P=0.012)$ [37]. Click et al. reviewed 74 articles with 301 patients addressing the role of apheresis in hypertriglyceridemic acute pancreatitis[38]. Seventy percent of patients received the procedures within the first $48 \mathrm{~h}$, and almost $85 \%$ required one-two sessions. The apheresis resulted in a mean triglycerides reduction of $85.4 \%$ $(P<0.001)$. Similar to the other findings, the authors concluded that further research, about the efficacy of apheresis in reducing the severity of acute pancreatitis, is needed[38]. Other strategies to decrease the hypertriglyceridemia levels are continuous insulin and heparin therapy, drugs that stimulate lipoprotein lipase activity and secondary chylomicron breakdown[39-42]. Patel recommends an insulin infusion of $0.1 \mathrm{IU} / \mathrm{kg} / \mathrm{h}$ co-administered with dextrose, to keep the blood glucose between 150-200 mg/dL[40]. Coskun et al. added to usual treatment insulin therapy in 12 patients with HAP[43]. Six patients had severe acute pancreatitis and eight presented diabetes mellitus. In all cases, following insulin therapy, the triglycerides decrease to less than $500 \mathrm{mg} / \mathrm{dL}$ in a mean time of three days[43]. This effect of insulin explains why patients with uncontrolled type one and two diabetes mellitus may develop HAP due to excessive lipolysis from the absence of insulin effect during diabetic ketoacidosis[44].

In an effort to prevent recurrence of the HAP in patients with severe hypertriglyceridemia, Chang et al. used prophylactic plasmapheresis[37]. Comparing six patients with severe hypertriglyceridemia and prophylactic plasmapheresis with six patients without this procedure the authors found that acute pancreatitis recurred in 0 vs. 3 cases $(P=0.046)[37]$. A very extreme attitude in a 42-years old female with eight episodes of HAP was described by Swedziak et al.[45]. They performed the exclusion of the distal common bile duct through a Roux-en-Y biliary bypass, using a 70-cm jejunum limb. During the six-month follow-up, there was no recurrence of acute pancreatitis, and the triglycerides serum value decreases from $993 \mathrm{mg} / \mathrm{dL}$ to $245 \mathrm{mg} / \mathrm{dL}[45]$.
We may conclude that HAP should be managed to respect all the general principles, also adding all the available resources to lower the serum triglycerides value, as early as possible in the acute setting and on long-term to prevent recurrences.

\section{Conflict of interest statement}

The authors report no conflict of interest.

\section{References}

[1] Baranyai T, Terzin V, Vajda A, Wittmann T, Czako L. [Acute pancreatitis caused by hypertriglyceridemia]. Orv Hetil 2010; 151(45): 1869-74. Hungarian.

[2] Senosiain Lalastra C, Tavio Hernandez E, Moreira Vicente V, Maroto Castellanos M, Garcia Sanchez MC, Aicart Ramos M, et al. [Acute hypertrygliceridemic pancreatitis]. Gastroenterol Hepatol 2013; 36(4): 274-9. Spanish.

[3] Valdivielso P, Ramirez-Bueno A, Ewald N. Current knowledge of hypertriglyceridemic pancreatitis. Eur J Intern Med 2014; 25(8): 68994.

[4] Scherer J, Singh V, Pitchumoni CS, Yadav D. Issues in hypertriglyceridemic pancreatitis - an update. J Clin Gastroenterol 2014; 48(3): 195-203.

[5] Toskes PP. Hyperlipidemic pancreatitis. Gastroenterol Clin North Am 1990; 19(4): 783-91.

[6] Durrington P. Dyslipidaemia. Lancet 2003; 362(9385): 717-31.

[7] Rosenson R. Approach to the patient with hypertriglyceridemia. UpToDate; 2016. [Online] Available from: http://www.uptodate. com/contents/approach-to-the-patient-with-hypertriglyceridemia?so urce $=$ search_result $\&$ search $=$ dyslipidemia\&selectedTitle $=1 \% 7 \mathrm{E} 150$ [Accessed on 20th May, 2016]

[8] Greenberg JA, Hsu J, Bawazeer M, Marshall J, Friedrich JO, Nathens A, et al. Clinical practice guideline: management of acute pancreatitis. Can J Surg 2016; 59(2): 128-40.

[9] Working Group IAP/APA Acute Pancreatitis Guidelines. IAP/APA evidence-based guidelines for the management of acute pancreatitis. Pancreatology 2013; 13: e1-15.

[10] Negoi I, Paun S, Hostiuc S, Moldoveanu A, Beuran M. Surgical intervention in acute pancreatitis: timing and coordination are the key. In: WSES-Critical Surgical Abdomen Consensus Conference 2016; 2016 Jul 21-26; Dublin, Ireland.

[11] Qiu L, Sun RQ, Jia RR, Ma XY, Cheng L, Tang MC, et al. Comparison of existing clinical scoring systems in predicting severity and prognoses of hyperlipidemic acute pancreatitis in Chinese patients: a retrospective study. Medicine (Baltimore) 2015; 94(23): e957.

[12] Tai WP, Lin XC, Liu H, Wang CH, Wu J, Zhang NW, et al. A retrospective research of the characteristic of hypertriglyceridemic pancreatitis in Beijing, China. Gastroenterol Res Pract 2016; 2016: 6263095.

[13] Carr RA, Rejowski BJ, Cote GA, Pitt HA, Zyromski NJ. Systematic review of hypertriglyceridemia-induced acute pancreatitis: a more virulent etiology? Pancreatology 2016; 16(4): 469-76.

[14] Beuran M, Negoi I, Catena F, Sartelli M, Hostiuc S, Paun S. 
Laparoscopic transgastric versus endoscopic drainage of a large pancreatic pseudocyst. A case report. J Gastrointestin Liver Dis 2016; 25(2): 243-7.

[15] Martin JM, Stapleton RD. Omega-3 fatty acids in critical illness. Nutr Rev 2010; 68(9): 531-41.

[16] Yeh JH, Chen JH, Chiu HC. Plasmapheresis for hyperlipidemic pancreatitis. J Clin Apher 2003; 18(4): 181-5.

[17] Chen JH, Yeh JH, Lai HW, Liao CS. Therapeutic plasma exchange in patients with hyperlipidemic pancreatitis. World J Gastroenterol 2004; 10(15): 2272-4.

[18] Huang C, Liu J, Lu Y, Fan J, Wang X, Liu J, et al. Clinical features and treatment of hypertriglyceridemia-induced acute pancreatitis during pregnancy: a retrospective study. J Clin Apher 2016; 31(6): 571-8.

[19] Schwartz J, Winters JL, Padmanabhan A, Balogun RA, Delaney M, Linenberger ML, et al. Guidelines on the use of therapeutic apheresis in clinical practice-evidence-based approach from the writing committee of the American Society for Apheresis: the sixth special issue. J Clin Apher 2013; 28(3): 145-284.

[20] National Cholesterol Education Program (NCEP) Expert Panel on Detection, Evaluation, and Treatment of High Blood Cholesterol in Adults (Adult Treatment Panel III). Third Report of the National Cholesterol Education Program (NCEP) Expert Panel on Detection, Evaluation, and Treatment of High Blood Cholesterol in Adults (Adult Treatment Panel III) final report. Circulation 2002; 106(25): 3143 421.

[21] Berglund L, Brunzell JD, Goldberg AC, Goldberg IJ, Sacks F, Murad $\mathrm{MH}$, et al. Evaluation and treatment of hypertriglyceridemia: an endocrine society clinical practice guideline. J Clin Endocrinol Metab 2012; 97(9): 2969-89.

[22] Murphy MJ, Sheng X, MacDonald TM, Wei L. Hypertriglyceridemia and acute pancreatitis. JAMA Intern Med 2013; 173(2): 162-4.

[23] Kayatas SE, Eser M, Cam C, Cogendez E, Guzin K. Acute pancreatitis associated with hypertriglyceridemia: a life-threatening complication. Arch Gynecol Obstet 2010; 281(3): 427-9.

[24] Vandenbroucke L, Seconda S, Lassel L, Le Bouar G, Poulain P. [Acute pancreatitis induced by major hypertriglyceridemia during pregnancy. A case report]. J Gynecol Obstet Biol Reprod (Paris) 2009; 38(5): 436-9. French.

[25] Singh HK, Prasad MS, Kandasamy AK, Dharanipragada K. Tamoxifen-induced hypertriglyceridemia causing acute pancreatitis. $J$ Pharmacol Pharmacother 2016; 7(1): 38-40.

[26] Sakhri J, Ben Salem C, Harbi H, Fathallah N, Ltaief R. Severe acute pancreatitis due to tamoxifen-induced hypertriglyceridemia with positive rechallenge. JOP 2010; 11(4): 382-4.

[27] Artac M, Sari R, Altunbas H, Karayalcin U. Asymptomatic acute pancreatitis due to tamoxifen-induced severe hypertriglyceridemia in a patient with diabetes mellitus and breast cancer. J Chemother 2002; 14(3): 309-11.

[28] Wasan KM, Goss PE, Pritchard PH, Shepherd L, Palmer MJ, Liu $\mathrm{S}$, et al. The influence of letrozole on serum lipid concentrations in postmenopausal women with primary breast cancer who have completed 5 years of adjuvant tamoxifen (NCIC CTG MA.17L). Ann Oncol 2005; 16(5): 707-15.

[29] Subramaniam S, Zell JA, Kunz PL. Everolimus causing severe hypertriglyceridemia and acute pancreatitis. J Natl Compr Canc Netw
2013; 11(1): 5-9.

[30] Sun HY, Chang SY, Sheng WH, Chen MY, Hsieh SM, Tseng YT, et al. Incidence of acute pancreatitis in human immunodeficiency virus-positive patients with hypertriglyceridemia: is it really high? Pancreas 2012; 41(2): 283-9.

[31] Baranyai T, Terzin V, Vajda Á, Wittmann T, Czakó L. Hypertriglyceridemia causes more severe course of acute pancreatitis. Clin Lipidol 2012; 7(6): 731-6.

[32] Rashid N, Sharma PP, Scott RD, Lin KJ, Toth PP. Allcause and acute pancreatitis health care costs in patients with severe hypertriglyceridemia. Pancreas 2016; doi: 10.1097/ MPA.0000000000000704.

[33] Deng LH, Xue P, Xia Q, Yang XN, Wan MH. Effect of admission hypertriglyceridemia on the episodes of severe acute pancreatitis. World J Gastroenterol 2008; 14(28): 4558-61.

[34] Wu C, Ke L, Tong Z, Li B, Zou L, Li W, et al. Hypertriglyceridemia is a risk factor for acute kidney injury in the early phase of acute pancreatitis. Pancreas 2014; 43(8): 1312-6.

[35] Zeng Y, Wang X, Zhang W, Wu K, Ma J. Hypertriglyceridemia aggravates ER stress and pathogenesis of acute pancreatitis. Hepatogastroenterology 2012; 59(119): 2318-26.

[36] An F, Zhan Q, Xia M, Jiang L, Lu G, Huang M, et al. From moderately severe to severe hypertriglyceridemia induced acute pancreatitis: circulating miRNAs play role as potential biomarkers. PloS One 2014; 9(11): e111058.

[37] Chang CT, Tsai TY, Liao HY, Chang CM, Jheng JS, Huang WH, et al. Double filtration plasma apheresis shortens hospital admission duration of patients with severe hypertriglyceridemia-associated acute pancreatitis. Pancreas 2016; 45(4): 606-12.

[38] Click B, Ketchum AM, Turner R, Whitcomb DC, Papachristou GI, Yadav D. The role of apheresis in hypertriglyceridemia-induced acute pancreatitis: a systematic review. Pancreatology 2015; 15(4): 313-20.

[39] Monga A, Arora A, Makkar RP, Gupta AK. Hypertriglyceridemiainduced acute pancreatitis--treatment with heparin and insulin. Indian J Gastroenterol 2003; 22(3): 102-3.

[40] Patel AD. Hypertriglyceridemia-induced acute pancreatitis treatment with insutin and heparin. Indian J Endocrinol Metab 2012; 16(4): 671-2.

[41] Twilla JD, Mancell J. Hypertriglyceridemia-induced acute pancreatitis treated with insulin and heparin. Am J Health Syst Pharm 2012; 69(3): 213-6.

[42] Alagozlu H, Cindoruk M, Karakan T, Unal S. Heparin and insulin in the treatment of hypertriglyceridemia-induced severe acute pancreatitis. Dig Dis Sci 2006; 51(5): 931-3.

[43] Coskun A, Erkan N, Yakan S, Yildirim M, Carti E, Ucar D, et al. Treatment of hypertriglyceridemia-induced acute pancreatitis with insulin. Prz Gastroenterol 2015; 10(1): 18-22.

[44] Wolfgram PM, Macdonald MJ. Severe hypertriglyceridemia causing acute pancreatitis in a child with new onset type I diabetes mellitus presenting in ketoacidosis. J Pediatr Intensive Care 2013; 2(2): 7780.

[45] Szwedziak K, Muras K, Strzelczyk J. Roux-en-Y biliary by-pass a new approach in the treatment of hypertriglyceridemia induced recurrent acute pancreatitis. Clinical case study. Pol Przegl Chir 2013; 85(3): 137-40. 